\title{
A PARALLEL MONTE CARLO CODE FOR SIMULATING COLLISIONAL $N$-BODY SYSTEMS
}

\author{
Bharath Pattabiraman $^{1,2}$, Stefan Umbreit $^{1,3}$, Wei-Keng Liao ${ }^{1,2}$, Alok $^{\text {Choudhary }}{ }^{1,2}$, \\ VASSIliki Kalogera $^{1,3}$, GoKhan MemiK ${ }^{1,2}$, AND Frederic A. Rasio ${ }^{1,3}$ \\ ${ }^{1}$ Center for Interdisciplinary Exploration and Research in Astrophysics, Northwestern University, Evanston, IL, USA; bharath@u.northwestern.edu \\ ${ }^{2}$ Department of Electrical Engineering and Computer Science, Northwestern University, Evanston, IL, USA \\ ${ }^{3}$ Department of Physics and Astronomy, Northwestern University, Evanston, IL, USA \\ Received 2012 June 18; accepted 2012 November 14; published 2013 January 18
}

\begin{abstract}
We present a new parallel code for computing the dynamical evolution of collisional $N$-body systems with up to $N \sim 10^{7}$ particles. Our code is based on the Hénon Monte Carlo method for solving the Fokker-Planck equation, and makes assumptions of spherical symmetry and dynamical equilibrium. The principal algorithmic developments involve optimizing data structures and the introduction of a parallel random number generation scheme as well as a parallel sorting algorithm required to find nearest neighbors for interactions and to compute the gravitational potential. The new algorithms we introduce along with our choice of decomposition scheme minimize communication costs and ensure optimal distribution of data and workload among the processing units. Our implementation uses the Message Passing Interface library for communication, which makes it portable to many different supercomputing architectures. We validate the code by calculating the evolution of clusters with initial Plummer distribution functions up to core collapse with the number of stars, $N$, spanning three orders of magnitude from $10^{5}$ to $10^{7}$. We find that our results are in good agreement with self-similar core-collapse solutions, and the core-collapse times generally agree with expectations from the literature. Also, we observe good total energy conservation, within $\lesssim 0.04 \%$ throughout all simulations. We analyze the performance of the code, and demonstrate near-linear scaling of the runtime with the number of processors up to 64 processors for $N=10^{5}, 128$ for $N=10^{6}$ and 256 for $N=10^{7}$. The runtime reaches saturation with the addition of processors beyond these limits, which is a characteristic of the parallel sorting algorithm. The resulting maximum speedups we achieve are approximately $60 \times, 100 \times$, and $220 \times$, respectively.
\end{abstract}

Key words: galaxies: clusters: general - globular clusters: general - methods: numerical

Online-only material: color figures

\section{INTRODUCTION}

The dynamical evolution of dense star clusters is a problem of fundamental importance in theoretical astrophysics. Important examples of star clusters include globular clusters, spherical systems containing typically $10^{5}-10^{7}$ stars within radii of just a few parsecs, and galactic nuclei, even denser systems with up to $10^{9}$ stars contained in similarly small volumes, and often surrounding a supermassive black hole at the center. Studying their evolution is critical to many key unsolved problems in astrophysics. It connects directly to our understanding of star formation, as massive clusters are thought to be associated with major star formation episodes, tracing the star formation histories of their host galaxies. Furthermore, globular clusters can trace the evolution of galaxies over a significant cosmological time span, as they are the brightest structures with which one can trace the halo potential out to the largest radii, and they are very old, potentially even predating the formation of their own host galaxies. Unlike stars and planetary nebulae, globular clusters are not simply passive tracers of galaxy kinematics as their internal dynamics are affected by the galactic tidal field. Therefore, their internal properties and correlations with their host galaxies are likely to contain information on the merger history of galaxies and halos.

Dynamical interactions in dense star clusters play a key role in the formation of many of the most interesting and exotic astronomical sources, such as bright X-ray and gammaray sources, radio pulsars, and supernovae. The extreme local stellar densities, which can reach $\gtrsim 10^{6} \mathrm{pc}^{-3}$, give rise to complex dynamical processes: resonant stellar encounters, tidal captures, physical collisions, and high-speed ejections (Heggie $\&$ Hut 2003). The primary challenge in modeling dense clusters lies in the tight coupling of these processes and their scales as they influence and inform one another both locally, e.g., through close encounters or collisions on scales of $\sim 1-100 R_{\odot}$, or $10^{-8}-10^{-6} \mathrm{pc}$, and globally on the scale of the whole system through long-range gravitational interactions. Close binary interactions can occur frequently, every $\sim 10^{6}-10^{9} \mathrm{yr}$ depending on the cluster density, relative to the global cluster evolution timescale. Furthermore, in the time between close encounters, stellar particles_-single and binary-change their physical properties due to their internal nuclear evolution and due to mass and angular momentum transfer or losses. All these changes affect the rates of close encounters and couple to the overall evolution of the cluster.

Given these enormous ranges in spatial and temporal scales, simulating dense star clusters with a million stars or more is a formidable computational challenge. A thorough analysis of the scaling of the computational cost of direct $N$-body methods is presented in Hut et al. (1988). Although direct $N$-body methods are free of any approximations in the stellar dynamics, their steep $\propto N^{3}$ scaling has limited simulations to an initial $N \sim 10^{5}$ stars (Zonoozi et al. 2011; Jalali et al. 2012; Hurley \& Shara 2012). However, the number of stars in real systems like globular clusters and galactic nuclei can be orders of magnitude larger. Even for globular cluster size systems where the evolution is feasible to calculate with a direct $N$-body code, the total runtime "took the best part of a year" (Hurley \& Shara 2012, p. 2878) 
and statistical results have to rely on only a very few models. This is a problem given the significant inherent stochasticity of these systems, which affects even basic structural parameters (e.g., Heggie \& Giersz 2009; Trenti et al. 2010; Hurley \& Shara 2012). In order to draw statistically robust conclusions, a much larger number of realizations of massive star clusters has to be calculated, in addition to a wider range of initial conditions. It is clear that these requirements result in prohibitive runtimes for direct $N$-body codes.

Monte Carlo (MC) methods calculate the dynamical evolution of the cluster in the Fokker-Planck approximation, which applies when the evolution of the cluster is dominated by twobody relaxation, and the relaxation time is much larger than the dynamical time. In practice, further assumptions of spherical symmetry and dynamical equilibrium have to be made. The Hénon MC technique (Hénon 1971), which is based on orbit averaging, represents a balanced compromise between realism and speed. The MC method allows for a star-by-star realization of the cluster, with its $N$ particles representing the $N$ stars in the cluster. Integration is done on the relaxation timescale, and the total computational cost scales as $\mathcal{O}(N \log N)$ (Hénon 1971).

Our work here is based on the Hénon-type MC cluster evolution code CMC ("Cluster Monte Carlo"), developed over many years by Joshi et al. (2000, 2001), Fregeau et al. (2003), Fregeau \& Rasio (2007), Chatterjee et al. (2010), and Umbreit et al. (2012). CMC includes a detailed treatment of strong binary star interactions and physical stellar collisions (Fregeau \& Rasio 2007), as well as an implementation of single and binary star evolution (Chatterjee et al. 2010) and the capability of handling the dynamics around a central massive black hole (Umbreit et al. 2012).

In addition to $\mathrm{CMC}$, other $\mathrm{MC}$ codes have been developed recently that are based on the same orbit averaging technique. Apart from differences in how the stellar and binary process have been implemented, these codes mainly differ in how particles are advanced in time. The code of Freitag \& Benz (2001) uses an individual time step scheme, where each particle is advanced on its own, local relaxation timescale, while the code of Giersz (1998, with its newest version described in Giersz et al. 2011) uses a block time step scheme, where the cluster is divided into several radial zones, and particles are evolved on the average relaxation timescale of the corresponding zone. While they provide better adaptability to density contrasts, individual and block time step schemes are more difficult to parallelize efficiently in a distributed fashion. A shared time step scheme, as implemented in CMC, offers a greater degree of inherent parallelism (Joshi et al. 2001).

A typical simulation starting with $N \sim 10^{6}$ up to average cluster ages of 12 Gyr using CMC can be run on a modern desktop computer in a reasonable amount of time (days to weeks). However, given the scaling of computational cost, simulations of clusters with $N \gtrsim 10^{7}$ stars, e.g., nuclear star clusters or galactic nuclei, will still take a prohibitive amount of time. Scaling up to even larger number of stars becomes possible only through parallelization.

In this paper, we present in detail the latest version of CMC, which is capable of simulating collisional systems of up to $N \sim 10^{7}$. In Section 2, we take a look at the components of the serial code and summarize both its numerical and computational aspects. In Section 3, we describe the flow of the parallel code, elucidating how we designed each part to achieve optimal performance on distributed parallel architectures. In addition, we describe in the Appendix, an optional CMC feature that accelerates parts of the code using a general purpose graphics processing unit (GPU). We show a comparison of results and analyze the performance of the code in Section 4. Conclusions and lines of future work are discussed in Section 5.

\section{CODE OVERVIEW}

\subsection{Numerical Methods}

Starting with an initial spherical system of $N$ stars in dynamical equilibrium, we begin by assigning to each star a mass, a position, and a velocity (radial and transverse components) by sampling from a distribution function $f(E, J)$, where $E$ and $J$ are the orbital energy and angular momentum (e.g., Binney \& Tremaine 2008). We assign positions to the stars in a monotonically increasing fashion, so the stars are initially sorted by their radial position. The system is assumed to be spherically symmetric and hence we ignore the direction of the position vector and transverse velocity. Following initialization, the serial algorithm goes through the following sequence of steps iteratively over a specified number of time steps. Figure 1 shows the flowchart of our basic algorithm.

1. Potential calculation. The stars having been sorted by increasing radial positions in the cluster, the potential at a radius $r$, which lies between two stars at positions $r_{k}$ and $r_{k+1}$, is given by

$$
\Phi(r)=G\left(-\frac{1}{r} \sum_{i=1}^{k} m_{i}-\sum_{i=k+1}^{N} \frac{m_{i}}{r_{i}}\right),
$$

where $m_{i}$ is the mass and $r_{i}$ is the position of star $i$. It is sufficient to compute and store the potential $\Phi_{k}=\Phi\left(r_{k}\right)$ at radial distances $r_{k}(k=1, \ldots, N)$, i.e., at the positions of all stars. This can be done recursively as follows:

$$
\begin{gathered}
\Phi_{N+1}=0, \\
M_{N}=\sum_{i=1}^{N} m_{i}, \\
\Phi_{k}=\Phi_{k+1}-G M_{k}\left(\frac{1}{r_{k}}-\frac{1}{r_{k+1}}\right), \\
M_{k-1}=M_{k}-m_{k} .
\end{gathered}
$$

To get the potential $\Phi(r)$ at any other radius, one first finds $k$ such that $r_{k} \leqslant r \leqslant r_{k+1}$ and then computes:

$$
\Phi(r)=\Phi_{k}+\frac{1 / r_{k}-1 / r}{1 / r_{k}-1 / r_{k+1}}\left(\Phi_{k+1}-\Phi_{k}\right) .
$$

2. Time step calculation. Different physical processes are resolved on different timescales. We use a shared time step scheme where time steps for all the physical processes to be simulated are calculated and their minimum is chosen as the global time step for the current iteration. The time steps are calculated using the following expressions (see Fregeau \& Rasio 2007; Goswami et al. 2011; Chatterjee et al. 2010 for more details):

$$
T_{\text {rel }}=\frac{\theta_{\max }}{\pi / 2} \frac{\pi}{32} \frac{\left\langle v_{\text {rel }}\right\rangle^{3}}{\ln (\gamma N) G^{2} n\left\langle\left(M_{1}+M_{2}\right)^{2}\right\rangle},
$$




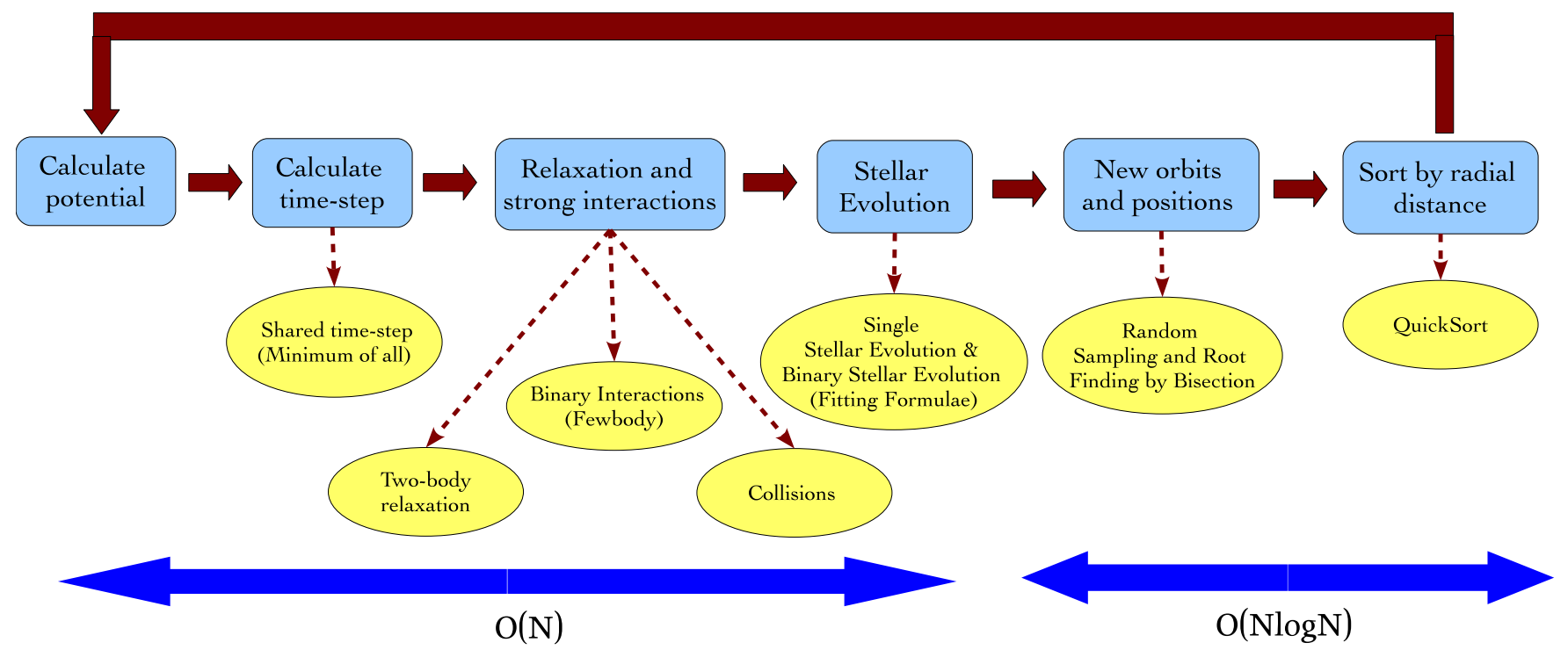

Figure 1. Flowchart of the CMC (Cluster Monte Carlo) code with the following steps. (1) Potential calculation-calculates the spherically symmetric potential. (2) Time step calculation — computes a shared time step used by all processes. (3) Relaxation and strong interactions-depending on the physical system type, performs two-body relaxation, strong interaction, or physical collision on every pair of stars. (4) Stellar evolution - evolves each star and binary using fitting formulae. (5) New positions and orbits—samples new positions and orbits for stars. (6) Sorting—sorts stars by radial position.

(A color version of this figure is available in the online journal.)

$$
\begin{gathered}
T_{\mathrm{coll}}^{-1}=16 \sqrt{\pi} n_{s}\left\langle R^{2}\right\rangle \sigma\left(1+\frac{G\langle M R\rangle}{2 \sigma^{2}\left\langle R^{2}\right\rangle}\right), \\
T_{\mathrm{bb}}^{-1}=16 \sqrt{\pi} n_{b} X_{\mathrm{bb}}^{2}\left\langle a^{2}\right\rangle \sigma\left(1+\frac{G\langle M a\rangle}{2 \sigma^{2} X_{\mathrm{bb}}\left\langle a^{2}\right\rangle}\right), \\
T_{\mathrm{bs}}^{-1}=4 \sqrt{\pi} n_{s} X_{\mathrm{bs}}^{2}\left\langle a^{2}\right\rangle \sigma\left(1+\frac{G\langle M\rangle\langle a\rangle}{\sigma^{2} X_{\mathrm{bs}}\left\langle a^{2}\right\rangle}\right), \\
T_{\mathrm{se}}=0.001 M\left(\frac{T_{\mathrm{prev}}}{\Delta m_{\mathrm{se}}}\right)
\end{gathered}
$$

where $T_{\text {rel }}, T_{\text {coll }}, T_{\mathrm{bb}}, T_{\mathrm{bs}}$, and $T_{\mathrm{se}}$ are the relaxation, collision, binary-binary, binary-single, and stellar evolution time steps respectively. Here $\theta_{\max }$ is the maximum angle of deflection of the two stars undergoing a representative two-body encounter, $v_{\text {rel }}$ is their relative velocities and $n$ is the local number density of stars, $n_{s}$ and $n_{b}$ are the number densities of single and binary stars, respectively, $\sigma$ is the one-dimensional velocity dispersion, and $a$ is the semimajor axis. $X_{\mathrm{bb}}$ and $X_{\mathrm{bs}}$ are parameters that determine the minimum closeness of an interaction to be considered a strong interaction. $M$ is the total mass of the cluster, $T_{\text {prev }}$ is the previous time step, and $\Delta m_{\mathrm{se}}$ is the mass loss due to stellar evolution.

The value of $T_{\text {rel }}$ is calculated for each star and the minimum is taken as the value of the global relaxation time step. We use sliding averages over the neighboring 10 stars on each side to calculate the mean quantities shown in $\langle\ldots\rangle$ in Equation (7). The other three time steps, $T_{\text {coll }}, T_{\mathrm{bb}}$, and $T_{\mathrm{bs}}$ are averaged over the central 300 stars as in Goswami et al. (2011). These choices provide a good compromise between accuracy and computational speed. Once these five time steps are calculated, the smallest one is chosen as the time step for the current iteration.

3. Relaxation and strong interactions (dynamics). Depending on the physical system type, one of the following three operations is performed on each pair of stars. (1) Two-body relaxation is applied based on an analytic expression for a representative encounter between two nearestneighbor stars. (2) A binary interaction (binary-binary or binary-single) is simulated using Fewbody, an efficient computational toolkit for evolving small- $N$ dynamical systems (Fregeau et al. 2004). Fewbody performs a direct integration of Newton's equations for three or four bodies using the eighth-order Runge-Kutta Prince-Dormand method. (3) A stellar collision is treated in the simple "sticky sphere" approximation, where two bodies are merged whenever their radii touch, and all properties are changed correspondingly (Fregeau \& Rasio 2007).

4. Stellar evolution. We use the single stellar evolution (Hurley et al. 2000) and binary stellar evolution (Hurley et al. 2002) routines, which are based on analytic functional fits to theoretically calculated stellar evolution tracks, to simulate the evolution of single and binary stars (Chatterjee et al. 2010).

5. New orbits computation. Consistent with the changes in the orbital properties of the stars following the interactions they undergo, new positions and velocities are assigned for orbits according to the new energy and angular momentum. Then, a new position is randomly sampled according to the amount of time the star spends near any given point along the orbit. Since this step represents a major computational bottleneck, we provide some details here.

We start by finding the pericenter and apocenter distances of the star's new orbit. Given a star with specific energy $E$ and angular momentum $J$ moving in the gravitational potential $\Phi(r)$, its rosette-like orbit $r(t)$ oscillates between two extreme values, $r_{\min }$ and $r_{\max }$, which are roots of

$$
Q(r)=2 E-2 \Phi(r)-J^{2} / r^{2}=0 .
$$

Since we only store the potential values at the positions of the stars, this equation cannot be analytically solved before determining the interval in which the root lies. In other words, we need to determine $k$ such that 
$Q\left(r_{k}\right)<0<Q\left(r_{k+1}\right)$. We use the bisection method to do this. Once the interval is found, $\Phi$, and thus $Q$, can be computed analytically in that interval, and so can $r_{\min }$ and $r_{\max }$.

The next step is to select a new radial position for the star between $r_{\max }$ and $r_{\min }$. The probability is equal to the fraction of time spent by the star in $d r$, i.e.,

$$
f(r)=\frac{d t}{T}=\frac{d r /\left|v_{\mathrm{r}}\right|}{\int_{r_{\min }}^{r_{\max }} d r /\left|v_{\mathrm{r}}\right|},
$$

where the radial velocity $v_{r}=[Q(r)]^{1 / 2}$. We use the von Neumann rejection technique to sample a position according to this probability. We take a number $F$ which is everywhere larger than the probability distribution $f(r)$. Then we draw two random numbers $X$ and $X^{\prime}$ and compute

$$
\begin{gathered}
r_{0}=r_{\min }+\left(r_{\max }-r_{\min }\right) X, \\
f_{0}=F X^{\prime} .
\end{gathered}
$$

If the point $\left(f_{0}, r_{0}\right)$ lies below the curve, i.e., if $f_{0}<f\left(r_{0}\right)$, we take $r=r_{0}$ as the new position; otherwise we reject it and draw a new point in the rectangle with a new pair of random numbers. We repeat this until a point below the curve is obtained. In our code, a slightly modified version of the method is used, since $f(r)=1 /\left|v_{r}\right|$ becomes infinite at both ends of the interval. A detailed description can be found in Joshi et al. (2000).

6. Sort stars by radial distance. This step uses the Quicksort algorithm (Hoare 1961) to sort the stars based on their new positions. Sorting the stars is essential to determine the nearest neighbors for relaxation and strong interactions, and also for computing the gravitational potential.

7. Diagnostics, energy conservation, and program termination. These involve the computation of diagnostic values such as half-mass radius, core radius, number of core stars, etc. that control program termination. In addition, corrections are made to the kinetic energy of each particle that account for the fact that the new orbit has been calculated in the old potential from the previous time step. This is done by adjusting the stellar velocities according to the mechanical work done on the stellar orbit by the time varying potential. We mainly follow the procedure in Stodolkiewicz (1982) except that we apply the correction to the velocity components such that the ratio $v_{r} / v_{t}$ is preserved. See Fregeau \& Rasio (2007) for more details. Since these minor calculations are scattered throughout various places in the code, they are not explicitly shown in the flowchart (Figure 1).

\subsection{Time Complexity Analysis}

In addition to the flow of the code, Figure 1 also shows the time complexity for each of the above steps. The time steps, $T_{\text {coll }}, T_{\mathrm{bb}}$, and $T_{\mathrm{bs}}$ are averaged over a fixed number of central stars, whereas $T_{\mathrm{se}}$ is a simple factor of the previous time step, and hence are $\mathcal{O}(1)$ operations. The calculation of $T_{\text {rel }}$ for a single star involves averaging over a fixed number of neighboring stars and hence has constant time complexity, $\mathcal{O}(1)$. As this is done for all $N$ stars to estimate their individual time steps from which the minimum is chosen, the time step calculation scales as $\mathcal{O}(N)$. The effect of relaxation and strong interactions is calculated between pairs of stars that are radially nearest neighbors. Since these calculations involve constant time operations for each of the $N$ stars, the time complexity of the perturbation step is $\mathcal{O}(N)$. Stellar evolution operates on a star-by-star basis performing operations of constant time for a given mass spectrum, and hence also scales as $\mathcal{O}(N)$. Determination of new orbits for each star involves finding the roots of an expression on an unstructured one-dimensional grid using the bisection method. The bisection method scales as $\mathcal{O}(\log N)$ and as this is done for each star, this step has a time complexity of $\mathcal{O}(N \log N)$. The radial sorting of stars using Quicksort has the same time complexity (Hoare 1961).

\section{PARALLELIZATION}

Achieving optimal performance of codes on parallel machines requires serial algorithms to be carefully redesigned, and hence, parallel algorithms are often very different than their serial counterparts and require a considerable effort to develop. The key to a successful algorithm is (1) good load balance, i.e., the efficient utilization of the available processing units and (2) minimal communication between these units. The communication cost depends directly on the choice of domain decomposition, i.e., the way in which work and data are partitioned into smaller units for processing in parallel. For example, a good domain decomposition scheme for achieving ideal load balance would be the distribution of stars (i.e., their data) evenly among the processors, assuming the computational cost for processing each star is similar. This will ensure that the workload is evenly balanced across processors given that they all perform the same number of operations, as in a Single Program, Multiple Data programming model. However, how such a decomposition would influence the need for communication between processing units is very specific to the algorithms used. In essence, a thorough knowledge of the algorithm and its data access patterns are necessary for designing any efficient parallel application.

\subsection{Data Dependencies and Parallel Processing Considerations}

While deciding upon the domain decomposition, we have to take into account any dependencies, i.e., the way the data are accessed by various parts of the application, as they may directly influence both the load balance and the communication costs between the processing units. A good parallel algorithm should distribute the workload in such a way that the right balance is struck between load balance and communication, resulting in optimal performance.

In CMC, the physical data of each star (mass, angular momentum, position, etc.) are stored in a structure, a grouping of data elements. The data for $N$ stars are stored an array of such structures. For a system with $p$ processors and $N$ initial stars, we will first consider the scenario where we try to achieve ideal load balance by naively distributing the data of $N / p$ stars to each processor. For simplicity, we will assume here that $N$ is divisible by $p$, and analyze the data dependencies in the various modules of CMC for this decomposition.

\section{Time step calculation.}

(a) For calculating the relaxation time of each star we need the local density, which is calculated using the masses of the 10 nearest neighboring stars on either side of the radially sorted list of stars. A parallel program would require communication between processors to exchange data of the neighboring stars that are at the extreme ends of the local array. 
(b) Calculation of the time step requires the computation of quantities in the innermost regions of the cluster, in particular the central density and the half-mass radius. If the particles are distributed across many processors, irrespective of the specific data partitioning scheme, identification of the particle up to which the enclosing stellar mass equals half the total mass of the cluster might require communication of intermediate results between adjacent data partitions, and also would introduce an inherent sequentiality in the code.

2. Relaxation and strong interactions (dynamics). For the perturbation calculation, pairs of neighboring stars are chosen. Communication might be needed depending on whether the number of stars in a processor is even or odd.

3. New orbits computation. To determine the new orbits of each star we use the bisection method which involves random accesses to the gravitational potential profile of the cluster. Since this data will be distributed in a parallel algorithm, communication will be needed for data accesses that fall outside the local subset.

4. Sorting. Putting the stars in order according to their radial positions naturally needs communication irrespective of the decomposition.

5. Potential calculation. The potential calculation, as explained in Section 2, is inherently sequential and requires communication of intermediate results.

6. Diagnostics and program termination. The diagnostic quantities that are computed on different computational units need to be aggregated before the end of the time step to check for errors or termination conditions.

\subsection{Domain Decomposition and Algorithm Design}

Based on the considerations in the previous section, we design the algorithms and decompose the data according to the following scheme so as to minimize communication costs, and at the same time not degrade the accuracy of the results.

Since the relaxation time step calculation procedure introduces additional communication cost irrespective of the choice of data partitioning, we modify it in the following way. Instead of using sliding averages for each star, we choose radial bins containing a fixed number of stars to calculate the average quantities needed for the time step calculation. We choose a bin size of 20 which gives a good trade off between computational speed and accuracy. We tested this new time step calculation scheme, and we did not find any significant differences compared to the previous scheme. In addition, we distribute the stars such that the number of stars per processor is a multiple of 20 (for the time step and density estimates) for the first $(p-1)$ processors and the rest to the last processor. Since 2 is a multiple of 20 , this removes any dependencies due to the interactions part as well. Our choice of data partitioning also ensures a good load balance as, in the worst case, the difference between the maximum and minimum number of stars among the processors could be at most 19 .

The gravitational potential $\Phi(r)$ is accessed in a complex, data-dependent manner as we use the bisection method to determine new stellar orbits. Hence, we do not partition it among the processors, but maintain a copy of it on all nodes. We also do the same for the stellar masses and positions, to remove the dependency in the potential calculation. This eliminates the communication required by the new orbits and potential calculations. However, it introduces the requirement to keep these data arrays synchronized at each time step and hence adds to the communication. We estimate the communication required for synchronization to be much less than what would be added by the associated dependencies without the duplicated arrays.

Most modules of the code perform computations in loops over the local subset of stars that have been assigned to the processor. Depending on the computation, each processor might need to access data from the local and duplicated arrays. While the local arrays can be accessed simply using the loop index, any accesses of the duplicated arrays (potential, position, or mass) require an index transformation. For instance, let us consider a simple energy calculation routine that calculates the energy of each star in the local array over a loop using the equation

$$
E_{i}=\Phi_{g i}+0.5\left(v_{r, i}^{2}+v_{t, i}^{2}\right)
$$

where $E_{i}, v_{r, i}$, and $v_{t, i}$ are the energy, radial velocity, and transverse velocity of star $i$ in the local array. The potential array having been duplicated across all processors, the potential at the position of the $i$ th star is $\Phi_{g i}$, where $g i$ is the global index given by the following transformation which directly follows from our data partitioning scheme explained above:

$g i= \begin{cases}i+i d\left\lfloor\left\lfloor\frac{N}{n_{m}}\right\rfloor \frac{1}{p}\right\rfloor n_{m}+i d n_{m} & \text { for } i d<\left\lfloor\frac{N}{n_{m}}\right\rfloor \bmod p, \\ i+i d\left\lfloor\left\lfloor\frac{N}{n_{m}}\right\rfloor \frac{1}{p}\right\rfloor n_{m}+\left\lfloor\frac{N}{n_{m}}\right\rfloor \bmod p n_{m} & \text { for } i d \geqslant\left\lfloor\frac{N}{n_{m}}\right\rfloor \bmod p .\end{cases}$

where $i d$ is the id of the processor that is executing the current piece of code, which ranges between 0 to $p-1, n_{m}$ is the number we would want the number of stars in each processor to be a multiple of, which as per our choice, is 20 , and the terms between $\lfloor\ldots\rfloor$ are rounded to the lowest integer.

\subsection{Parallel Flow}

The following gives an overview of the parallel workflow.

1. Initial partitioning of the star data and distribution of duplicated arrays (mass and radial positions).

2. Calculate potential.

3. Calculate timestep and perform interactions, stellar evolution, and new orbits computation.

4. Sort stars by radial position in parallel.

5. Redistribute/load-balance data according to domain decomposition.

6. Synchronize duplicated arrays (mass and radial positions).

Then the whole procedure is repeated starting from step 2 . The first step is to distribute the initial data among processors as per our data partitioning scheme mentioned in Section 3.2. This is done only once per simulation. This also includes the distribution of a copy of the duplicated arrays. In Section 2, we saw that the calculation of the potential is inherently sequential requiring communication, since it is calculated recursively starting from the outermost star and using the intermediate result to compute the potential of the inner stars. However, since now every processor has an entire copy of the potential, the positions, and mass arrays, it can calculate the potential independently. This does not give a performance gain since there is no division of workload, however, it nullifies the communication requirement. Performing interactions, stellar evolution, and new orbits calculation also do not require any communication whatsoever due to our choice of data partitioning and use of duplicated arrays. We use Sample Sort (Fraser \& McKellar 1970) as the parallel sorting algorithm (see Section 3.4). With a wise choice of parameters, Sample Sort can provide a 
near-equal distribution of particles among processors. However, since we require the data to be partitioned in a very specific way, following the sort, we employ a redistribution/loadbalancing phase to redistribute the sorted data as per our chosen domain decomposition. Sorting and redistribution are steps that naturally require the most communication. Before the beginning of the next time step, we synchronize the duplicated arrays on all processors which requires message passing. Some non-trivial communication is also required at various places in the code to collect and synchronize diagnostic values.

\subsection{Sorting}

The input to a parallel sorting algorithm consists of a set of data elements (properties of $N$ stars in our case), each having a key (radial positions in our case) based on which the data need to be sorted. An efficient parallel sorting algorithm should collectively sort data owned by individual processors in such a way that their utilization is maximum, and at the same time the cost of redistribution of data across processors is kept to a minimum. In order to implement a parallel sorting algorithm, there are a wide array of solutions to consider. Each of these solutions caters to a parallel application and/or in some cases a particular machine architecture/platform. In general, a parallel programmer should consider many different design decisions with careful consideration of the application characteristics. The proper assessment of application knowledge often can suggest which initial distributions of data among processors are likely to occur, allowing the programmer to implement a sorting algorithm that works effectively for that application.

The importance of load balance is also immense since the application's execution time is typically bound by the local execution time of the most overloaded processor. Since our choice of domain decomposition requires a fairly good load balance, our sorting algorithm should ensure that the final distribution of keys among processors closely agrees with our decomposition. This is a challenge since during their evolution, dense star clusters have a very strong density contrast, and stars are very unevenly distributed radially with a substantial number of stars in the high density core, and the rest in the extremely low density halo. A good parallel sorting algorithm for our application should be able to judge the distribution of keys so as to deliver almost equal amount of data in each processor at the end of the sorting phase.

Sample Sort is a splitter-based parallel sorting algorithm that performs a load balanced splitting of the data by sampling the global key set. This sampling helps judge the initial distribution of keys and accordingly perform the sort, hence resulting in a near-equal distribution of data among processors. Given $N$ data elements distributed across $p$ processors, Sample Sort consists of five phases, shown in Figure 2.

1. Sort local data. Each processor has a contiguous block of data in accordance with our data partition (close to $N / p$ in number, see Section 3.2). Each processor, in parallel, sorts this local block using sequential Quicksort.

2. Sampling. All $p$ processors, in parallel, uniformly sample $s$ keys to form a representative sample of the locally sorted block of data. These set of $p$ samples, of size $s$ from each processor, are collected on one designated processor. This aggregated array of samples represents the distribution of the entire set of keys.

3. Splitter selection. The combined sample key array is sorted, and keys at indices $s, 2 s, 3 s, \ldots,(p-1) s$ are selected as splitters and are broadcasted to all processors.
4. Exchange partitions. The positions of the $(p-1)$ splitter points in the local array are determined by each processor using binary search; this splits the local array into $p$ partitions. In parallel, each processor retains the $i$ th partition and sends the $j$ th partition to the $j$ th processor, i.e., each processor keeps one partition and distributes $(p-1)$ partitions. At the same time it receives one partition from every other processor. This might not be true always, particularly in cases of a poor choice of sample size $s$, some splitter points might lie outside the local data array and hence some processors might not send data to all $(p-1)$ processors but only a subset of them. However, for the current discussion we will assume a good choice of sample size is made.

5. Merge partitions. Each processor, in parallel, merges its $p$ partitions into a single list and sorts it.

One chooses a value for the sample size $s$ so as to sample the distribution of keys accurately, and hence this parameter varies depending on the distribution of keys as well the data size $N$. More comments on the choice of sample size can be found in Li et al. (1993).

Let us now try to derive the time complexity of Sample Sort. We assume a hypercube parallel architecture with cut-through routing, a message routing mechanism in which nodes immediately forward messages to subsequent ones as they arrive ${ }^{4}$. The local sort (Phase 1) requires $\mathcal{O}((N / p) \log (N / p))$ since there are close to $N / p$ keys per processor. The selection of $s$ sample keys (Phase 2, part 1 ) requires $\mathcal{O}(s)$ time. Collecting $s$ keys from $p$ processors on to one of the processors (Phase 2, part 2) is a single-node gather operation for which the time required is $\mathcal{O}(s p)$. The time to sort these $s p$ samples is $\mathcal{O}((s p) \log (s p))$, and the time to select $(p-1)$ splitters (Phase 3, part 1) is $\mathcal{O}(p)$. The splitters are sent to all the other processors (Phase 3, part 2) using an one-to-all broadcast which takes $\mathcal{O}(p \log p)$ time. To place the splitters in the local array (Phase 4, part 1) using binary search takes $\mathcal{O}(p \log (N / p))$. The all-to-all communication that follows (Phase 4, part 2) costs a worst case time of $\mathcal{O}(N / p)+\mathcal{O}(p \log p)$. The final step that merges and sorts the partitions would $\operatorname{cost} \mathcal{O}((N / p) \log (N / p))$ time. So the time complexity of the entire algorithm becomes

$$
\begin{aligned}
\mathcal{O} & \left(\frac{N}{p} \log \frac{N}{p}\right)+\mathcal{O}((s p) \log (s p)) \\
& +\mathcal{O}\left(p \log \frac{N}{p}\right)+\mathcal{O}(N / p)+\mathcal{O}(p \log p) .
\end{aligned}
$$

\subsection{Data Redistribution}

In theory, with a good choice of sample size, Sample Sort guarantees to distribute the particles evenly among processors within a factor of two ( $\mathrm{Li}$ et al. 1993). However, we would like to partition the data in such a way that each processor has close to $N / p$ elements, and at the same time being multiple of 20 . Since the final distribution of data among processors after Sample Sort is not deterministic, we include an additional communication phase to ensure the required domain decomposition is maintained.

We first calculate the global splitter points that would partition the entire data among processors as per our required data partitioning scheme. We then perform a parallel prefix reduction (MPI_Exscan; see Section 3.7), so each processor knows

\footnotetext{
4 This is faster than when the nodes wait until the entire message is received and stored before forwarding, also known as store-and-forward routing.
} 
Processor 1

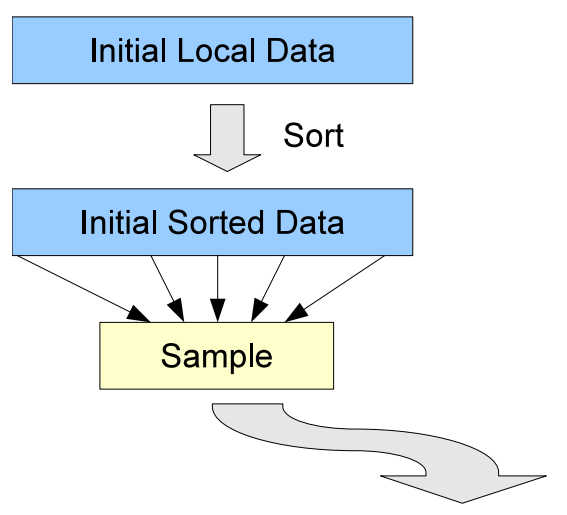

Processor $p$

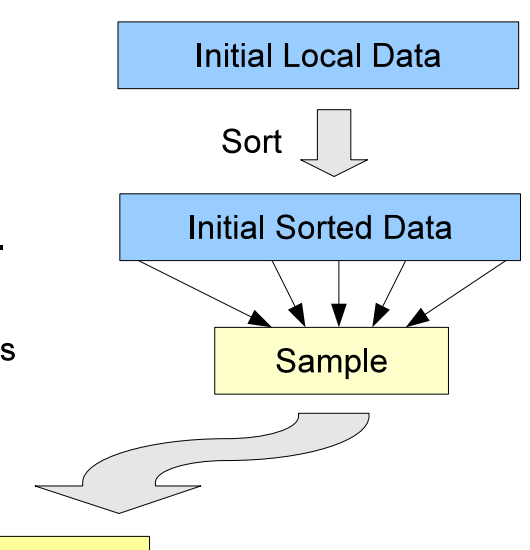

Local Samples



Combined Sample

Sort



Figure 2. Sample Sort algorithm.

(A color version of this figure is available in the online journal.)

the cumulative number of stars that ended up in the processors before it. Using this, it can calculate the range of global indices corresponding to the list of stars it currently holds. Now, each processor checks if any of the global splitter points other than its own map on to its local array, and if they do, it marks the corresponding chunk of data to be sent to the respective processor. Then, we perform an all-to-all communication, after which the data on each processor is sorted by simply rearranging the received chunks.

Let us consider an example where there are four processors and they receive $100,130,140$, and 80 stars, respectively, after the sorting phase. For a total of 450 stars to be divided among four processors, the expected distribution would be 120 , 120,100 , and 110 stars, respectively, as per our scheme. The corresponding global splitter points would be 120, 240, and
340. By performing the prefix reduction on the received number of stars, processor 3 , for instance, knows there are in total 230 stars in processors 1 and 2 put together. Since it received 140 stars after sorting, it also calculates that it has stars with indices between 231 and 370. Now, two global splitter points, i.e., 240 and 340 of processors 2 and 4 lie within this index range, and hence the corresponding stars, i.e., 231-240 and 341-370 need to be sent to processors 2 and 4, respectively. These data chunks are exchanged by performing an all-to-all communication, followed by a rearrangement if required.

\subsection{Parallel Random Number Generation}

The accuracy of results of a parallel MC code depends in general on both the quality of the pseudo-random number 
generators (PRNGs) used and the approach adopted to generate them in parallel. In our case we need to sample events with very low probability, such as physical collisions between stars or binary interactions, which makes it necessary for the generated random numbers to be distributed very evenly. More specifically, given $N_{r}$ random numbers uniformly distributed in the interval $(0,1)$, it would be preferable to have one random number in each sub-interval of size $1 / N_{r}$. A special class of random number generators for which this property holds in even higher dimensions are the maximally equidistributed generators and we choose here the popular and fast combined Tausworthe linear feedback shift register (LFSR) PRNG in L'Ecuyer (1999).

PRNGs use a set of state variables which are used to calculate random numbers. Every time a random number is generated, the values of these states are updated. A PRNG can be initialized to an arbitrary starting state using a random seed. When initialized with the same seed, a PRNG will always produce the same exact sequence. The maximum length of the sequence before it begins to repeat is called the period of the PRNG. The combined Tausworthe LFSR PRNG we are using here has a period of $\approx 2^{113}$ (L'Ecuyer 1999).

While generating random numbers in parallel, special care has to be taken to ensure statistical independence of the results calculated on each processor. For instance, to implement a parallel version, we could simply allocate separate state variables for the PRNGs on each processor and initialize them with a different random seed. However, choosing different seeds does not guarantee statistical independence between these streams.

An efficient way to produce multiple statistically independent streams is to divide a single random sequence into subsequences, with their starting states calculated using jump functions (Collins 2008). Taking a starting seed and a jump displacement, $D$, as inputs, jump functions can very quickly generate the $D$ th state of the random sequence. We use this method to generate multiple starting states, one for each processor, by repeatedly applying the jump function and saving intermediate results. The jump displacement is chosen as the maximum number of random numbers each processor might require for the entire simulation while still providing for a sufficiently large number of streams. Based on that we choose $D=2^{80}$.

\subsection{Implementation}

CMC is written in $\mathrm{C}$, with some parts in Fortran. We use the Message Passing Interface (MPI) library (Lusk et al. 1996) to handle communication. The MPI standard is highly portable to different parallel architectures, and available on practically every supercomputing platform in use today. The most common MPI communication calls (see Gropp et al. 1994 for a detailed description) used in our code are as follows.

\section{MPI_Allreduce/MPI_Reduce}

MPI_Reduce combines the elements of a distributed array by cumulatively applying a user specified operation as to reduce the array to a single value. For instance, when the operation is addition then the resulting value is the sum of all elements. MPI_Allreduce is MPI_Reduce with the difference that the result is distributed to all processors. The call is used in the following parts of the code.

(a) Diagnostics and program termination. Accumulating diagnostic quantities such as the half-mass radius, $r_{h}$, and the core radius, $r_{c}$. (b) Time step calculation. To find the minimum time step of all stars across processors.

(c) Sorting and data redistribution. Since stars are created and lost throughout the simulation, $N$ is not a constant and changes during a time step. It is calculated during the sorting step by summing up the local number of stars on each processor.

\section{MPI_Allgather/MPI_Gather}

In MPI_Gather each process sends the contents of its local array to the root, or master, process, which then concatenates all received arrays into one. In MPI_Allgather this concatenated array is distributed to all nodes. The calls are used in the following parts of the code.

(a) Synchronization of duplicated arrays, i.e., $\Phi(r)$ and the stellar masses.

(b) Sorting and data redistribution. To gather samples contributed by all processors on to a single node. See Section 3.4 for details.

\section{MPI_Alltoall}

In MPI_Alltoall the send and receive array is divided equally into $p$ sub-arrays, where $p$ is the number of processors. The position of each sub-array within the send or receive array determines to or from which processor the data are sent or received, respectively. MPI_Alltoall is only used in "Sorting and data redistribution." See Section 3.4 for details.

\section{MPI_Bcast}

In MPI_Bcast an array is sent from the root, or master, node to all other processes.

Used in "Sorting and data redistribution" to communicate the new splitter points from a single specified processor to all other.

5. MPI_Scan/MPI_Exscan

MPI_Scan essentially carries out a reduction as in MPI_Allreduce except that processor $i$ receives the result of the reduction over the data of processors 0 to $i$. In MPI_Exscan the data are reduced over processors 0 to $i-1$. MPI_Scan/Exscan is used in Sorting and data redistribution. See Section 3.5 for details.

We also use a number of optimizations for the MPI communication calls. Some examples include using MPI derived datatypes for data packing and sending, and combining multiple parallel reduction calls for the diagnostic quantities by packing all the data into a single buffer and performing the reduction together using a single call which is more efficient. However, the overlapping of communication calls with computation we did not explore so far, but intend to do so in the future.

\section{RESULTS}

All our test calculations were carried out on Hopper, a Cray XE6 supercomputer at NERSC ${ }^{5}$ that has a peak performance of 1.28 Petaflops, 153,216 processor cores for running scientific applications, 212 TB of memory, and 2 Petabytes of online disk storage.

\subsection{Accuracy and Reproducibility}

In the parallel version, since we use several different random sequences within one simulation, the way random numbers are assigned to stars is different from the serial version. This

\footnotetext{
5 http://www.nersc.gov/
} 


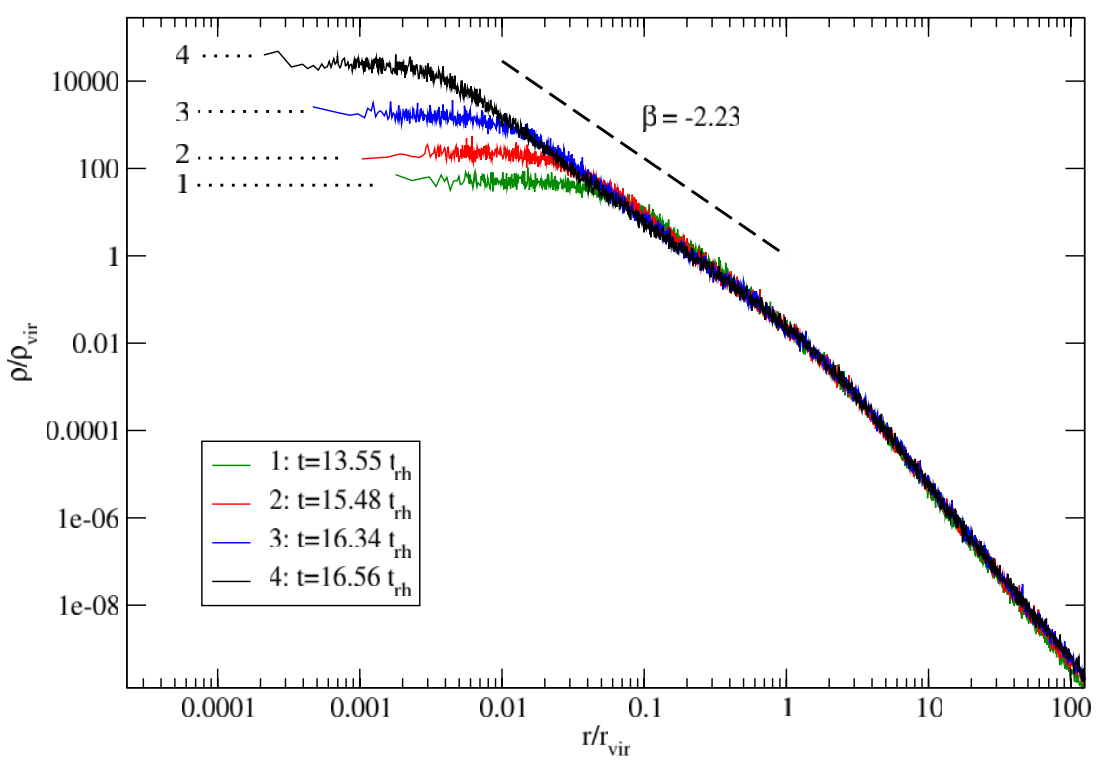

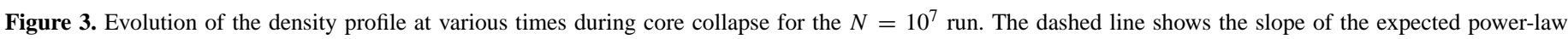
density profile (Heggie \& Stevenson 1988; Cohn 1980).

(A color version of this figure is available in the online journal.)

would introduce in a problem of inconsistency in the results between serial and parallel runs, leaving us with no simple way to verify the correctness of the results of our parallel version. We tackle this problem by changing the serial version such that it uses the same mapping of random number streams to stars as followed in the parallel version. This emulation allows us to compare the results of the parallel version with that of the serial version. However, note that parallel runs with different numbers of processors will result in a different mapping of streams to stars, making a separate serial run necessary to compare results for each case.

We ran test simulations for 50 time steps with the parallel and serial versions, using or emulating up to a few processors, respectively. Initially, the clusters had $N=10^{5}, 10^{6}$, and $10^{7}$ stars with positions and velocities distributed according to a Plummer model. By comparing the positions and masses of every star in the cluster at the end of the simulations, we found that the parallel and corresponding serial results were matching accurately down to the last significant digit (all variables are in double precision). We also compared a few diagnostic quantities, such as the core radius, and core density, and they were matching as well, except for the last four significant digits. This slight loss in accuracy is due to the MPI_Reduce calls, which perform cumulative operations (sum, max, min, etc.) on data distributed among the processors. This introduces different round-off errors since one does not have control over the order in which the data aggregation is done.

\subsection{Comparison to Theoretical Predictions}

In order to verify that our code reproduces well-known theoretical results, we calculate the evolution of single-mass Plummer spheres (Binney \& Tremaine 2008) until core collapse (without any binaries or stellar evolution). With $10^{5}, 10^{6}$, and $10^{7}$ stars, this is the first time that collisional $N$-body simulations covering three orders of magnitude up to $N=10^{7}$ stars have been carried out. We used 128, 256, and 1024 processors for these runs, respectively, which deliver peak performance for the three cases (see Section 4.3). The wall clock runtimes for these simulations were 11.4 minutes, $1.17 \mathrm{hr}$, and $12.8 \mathrm{hr}$, respectively.
One remarkable property realized early on is that the cluster evolution proceeds asymptotically in a self-similar fashion, that is, the cluster density profiles differ only in scale and normalization at late times (e.g., Cohn 1980; Binney \& Tremaine 2008). This can be clearly seen in Figure 4, where we plot the density profile of the cluster with $N=10^{7}$ at various times during its evolution to core collapse. For each profile we observe a well-defined core and a power-law density profile, $\rho \propto r^{-\beta}$, with $\beta \approx 2.3$. This is only slightly larger than the value $\beta=2.23$ usually quoted in the literature (first obtained by Cohn 1980). The slight discrepancy, however, arises probably because the density profiles in Figure 3 were taken at a time when core collapse has not yet proceeded far enough.

Figure 4 shows the evolution of the core radius, $r_{\mathrm{c}}(t) / r_{\mathrm{h}}(t)$, as well as the core density, $\rho_{c}(t)$, for the models with $N=10^{5}, 10^{6}$, and $10^{7}$ stars. We use the notation $r_{c}$ for the density-weighted core radius (Casertano \& Hut 1985) and $\rho_{c}$ for the core density, defined as

$$
\rho_{c}=\frac{\sum_{i}\left[\rho_{i}\right]^{2}}{\sum_{i} \rho_{i}},
$$

where $\rho_{i}$ is the sixth-order density estimator around the $i$ th star (Casertano \& Hut 1985). The core density $\rho_{c}$ is expressed in units of $M_{c} r_{\mathrm{vir}}^{-3}$, where $M_{c}$ is the total cluster mass and $r_{\mathrm{vir}}$ is the virial radius, defined as $G M_{c}^{2} /\left(2 E_{0}\right)$ with $E_{0}$ the initial total gravitational energy of the cluster and $G$ the constant of gravity. One can immediately see that all three clusters reach core collapse at similar times, with $t=t_{\mathrm{cc}} \simeq 17.4 t_{\mathrm{rh}}, 16.7 t_{\mathrm{rh}}$, and $16.6 t_{\mathrm{rh}}$, respectively, where $t_{\mathrm{rh}}$ is the initial half-mass relaxation time defined as (Spitzer 1987)

$$
t_{r h}=\frac{0.138 N}{\ln (\gamma N)}\left(\frac{r_{h}^{3}}{G M}\right)^{1 / 2}
$$

with $\gamma$ in the Coulomb logarithm chosen to be 0.1 . Thus, the core-collapse times are not only in very good agreement with previously published results that all lie between 15 and 18 $t_{\text {rh }}$ (see, e.g., Freitag \& Benz 2001 for an overview), but also confirm that our code can reproduce the scaling of $t_{\mathrm{cc}}$ with $t_{\mathrm{rh}}$ 




Figure 4. Evolution of an isolated Plummer model showing the ratio of core radius to half-mass radius, $r_{\mathrm{c}} / r_{\mathrm{h}}$ (top), and the core density, $\rho_{c}$ (bottom). Time is in initial half-mass relaxation times. The various lines represent different particle numbers, $N=10^{5}$ (dashed), $10^{6}$ (dotted), $10^{7}$ (solid).

(A color version of this figure is available in the online journal.)

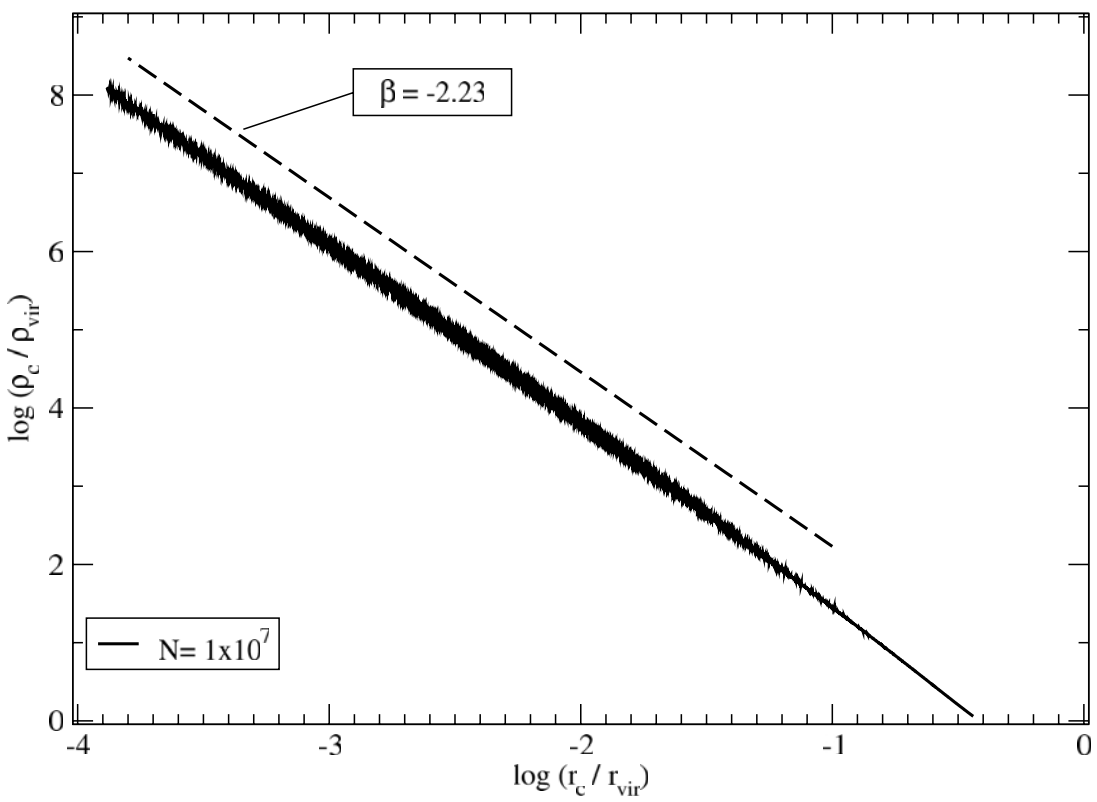

Figure 5. Core density as a function of $r_{c} / r_{\mathrm{vir}}$. The density is expressed in units of $\rho_{\mathrm{vir}}=M_{c} r_{\mathrm{vir}}^{-3}$. The dashed line shows the power-law slope expected for a cluster close to core collapse based on the self-similarity of the cluster evolution in that regime (see, e.g., Binney \& Tremaine 2008). In our simulation this regime appears to be reached for $r_{c} / r_{\mathrm{vir}} \lesssim 3 \times 10^{-4}$, and a power-law fit to the core density in this range results in a slope of -2.24 .

within $\approx 10 \%$ over three orders of magnitude in $N$. The scaling behavior becomes even better, with deviations $<1 \%$, if only the runs with $N \geqslant 10^{6}$ are considered. The larger deviation in $t_{\text {cc }}$ between the $N=10^{5}$ run and the other two are probably because of the larger stochastic variations in low $N$ runs.

Another consequence of the self-similarity of the cluster evolution to core collapse is that $-\beta \sim \log \left(\rho_{c}(t)\right) / \log \left(r_{\mathrm{c}}(t)\right)$ (Binney \& Tremaine 2008), which means that the decrease in $r_{\mathrm{c}}$ leads to an increase in $\rho_{\mathrm{c}}$ at a rate that is related to the shape of the density profile. Indeed, from Figure 4 one can see that the shape of $\rho_{\mathrm{c}}(t)$ mirrors the shape of $r_{\mathrm{c}}(t)$ as expected. Even more so, from Figure 5, we find that the powerlaw slope of $\rho_{c}\left(r_{c}\right)$ becomes $\beta=-2.24$ close to core collapse $\left(r_{c} / r_{\text {vir }}<3 \times 10^{-4}\right)$, which is in excellent agreement with the corresponding $\beta=-2.23$ found by Cohn (1980). It is worth noting that $\beta$ slowly changes with $r_{c}$, increasing from around $\beta=-2.5$ and eventually converging to $\beta=-2.24$, which is also reflected in the power-law slopes of the density profiles we obtain from our simulations for increasing times (Figure 3).

Apart from the self-similar behavior, we also find that there is very little mass loss $(\lesssim 1 \%)$, and hence very little energy is carried away by escaping stars, in agreement with theoretical expectations (e.g., Lightman \& Shapiro 1978). Finally, we find that our code conserves total energy to better than $0.04 \%$ throughout the entire simulation.

\subsection{Performance Analysis}

We tested our parallel code for three cluster models with $N=10^{5}, 10^{6}$, and $10^{7}$ stars, with an initial Plummer density 




Figure 6. Scaling of the wall clock time with the number of processors, $p$, for a parallel simulation of up to 50 time steps. The various lines represent different particle numbers (see the legend). We observe a near-linear scaling for up to 64 processors.

(A color version of this figure is available in the online journal.)

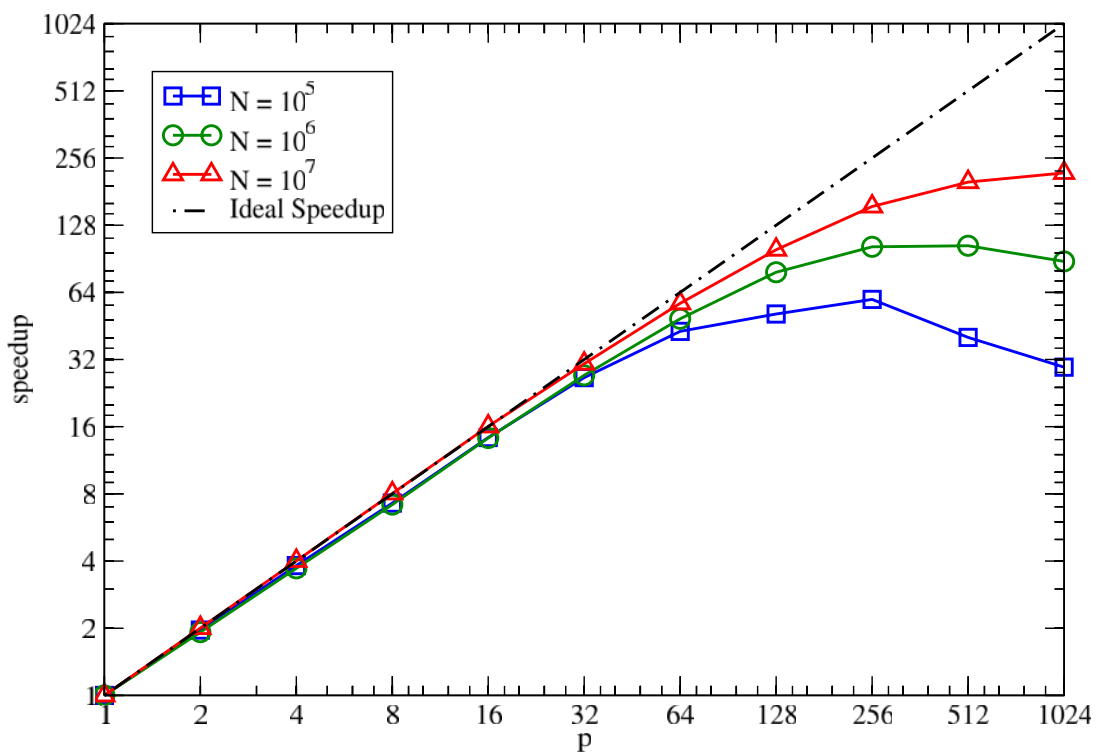

Figure 7. Speedup of our parallel code as a function of the number of processors, $p$. The various lines represent different particle numbers (see the legend). The straight line represents the ideal speedup, which is the case when the speedup equals $p$. We observe that for all three cases, the speedup closely follows the ideal speedup line for up to 64 processors.

(A color version of this figure is available in the online journal.)

profile. We used 1-1024 processors and measured the total time taken, and time taken by various parts of the code for up to 50 time steps. For the sample size $s$ in the Sample Sort algorithm, we chose 128,256 , and 1024, respectively, for the three $N$ values.

The timing results are shown in Figure 6 and a corresponding plot of the speedups in Figure 7. These results do not include the time taken for initialization. We can see that the speedup is nearly linear up to 64 processors for all three runs, after which there is a gradual decrease followed by saturation. For the $N=10^{5}$ and $10^{6}$ case, we also note a dip after the saturation, also expected for the $N=10^{7}$ case for a larger number of processors than we consider here. We also see that the number of processors for which the speedup peaks is different for each value of $N$, and gradually increases with $N$. The peak is seen at 256 processors for the $N=10^{5}$ run, somewhere between 256 and 512 for the $N=10^{6}$ run, and 1024 for the $N=10^{7}$ run. The maximum speedups observed are around $60 \times, 100 \times$, and $220 \times$ for the three cases, respectively.

Figure 8 shows the scaling of the time taken by various modules of our code for the $N=10^{6}$ run. One can observe that the dynamics, stellar evolution, and orbit calculation modules achieve perfectly linear scaling. The ones that do not scale as well are sorting and the "rest," which includes diagnostics, potential calculation, and time step calculation. As the number of processors increase, the linear scaling of the former three parts of the code reduces their time to very small values, in turn letting the parts that do not scale well dominate the runtime. This 




Figure 8. Time taken by the various parts of the code for a parallel run with $N=10^{6}$ stars. The various lines represent the different modules of the algorithm (see the legend). The module denoted by "Rest" is the cumulative time taken by diagnostics, potential calculation, and time step calculation steps. One can observe that the dynamics, stellar evolution, and new orbits computation modules scale linearly whereas the other two exhibit relatively poor scaling beyond 64 processors.

(A color version of this figure is available in the online journal.)

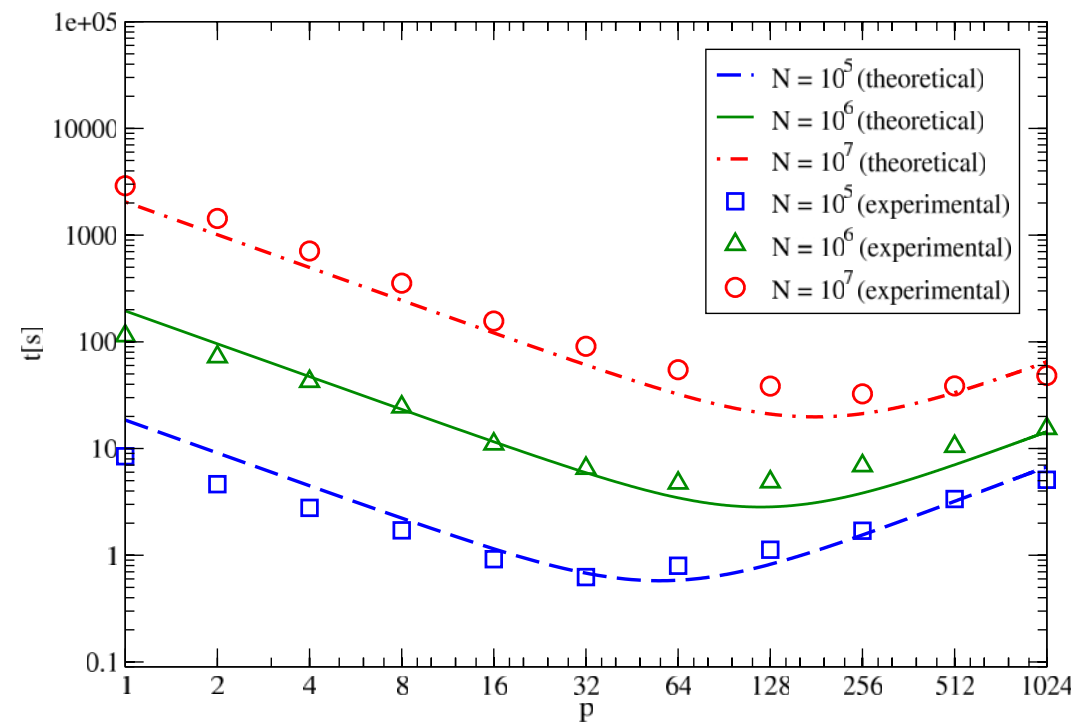

Figure 9. Time taken by the sorting routine of the parallel code plotted against the theoretical time complexity of Sample Sort based on Equation (18) for various values of $N$. Appropriate proportionality constants were used based on the data size transported during the all-to-all communication phase of Sample Sort (see Section 3.4).

(A color version of this figure is available in the online journal.)

is the reason for the trend observed in the total execution time and speedup plots. We can also particularly see that the time taken by sorting starts to increase after reaching a minimum, and this explains a similar observation in the previous plots as well.

Figure 9 shows the experimental timing results of our sorting step for the three $N$ values plotted against the theoretical values calculated using Equation (18). Since the entire star data are communicated during the all-to-all communication phase of the sort and not just the keys, and a data size of a single star is 46 times greater than that of a single key in our code, we multiply the $\mathcal{O}(N / p)$ term of Equation (18) with a proportionality constant of 46 . We see that for all three cases, the expected time linearly decreases, reaching a minimum after which it increases. This implies that for every data size, there is a threshold for the number of processors that would achieve optimal performance beyond which it will worsen. The main reason is that, as the number of processors increases, smaller amounts of data are distributed across many processors, which makes the communication overhead dominant. In addition, the number of samples to be accumulated and sorted on a single node (Phases 2 and 3 of Sample Sort; see Section 3.4) increases with the number of processors, and hence this phase tends to become a bottleneck as well. From Figure 9, we also see that our implementation very closely follows the trend predicted by the theoretical complexity formula. In addition, the number of processors at which maximum performance is attained match fairly closely as well.

The other parts of the code that do not scale well include diagnostics, potential calculation, and time step calculation. The computation of diagnostics and program termination related quantities require a number of collective communication 
calls to aggregate data across processors, and this is difficult to parallelize efficiently. However, there might be potential to improve this scaling by interleaving the collective communication calls with computation. While the time step calculation is embarrassingly parallel and can be expected to scale well, the time taken for potential calculation would remain constant irrespective of the number of processors used since every processor computes the entire potential array, and has little scope for optimization. A thorough profiling of the poorly scaling parts is necessary in the future to identify the dominant among these bottlenecks, and prioritize optimization steps.

We noted earlier that the total execution time followed a very similar trend as sorting, however, the places at which the minimum execution time was observed are not the same, butshifted to the right. We can now infer this to be a result of the linearly scaling parts of the code pushing these points to the right until the relatively poorly scaling parts dominate the runtime.

\section{CONCLUSIONS}

We presented a new parallel code, CMC, for simulating collisional $N$-body systems with up to $N \sim 10^{7}$. In order to maintain a platform-independent implementation, we adopt the MPI library for communication. The parallelization scheme uses a domain decomposition that guarantees a near-equal distribution of data among processors to provide a good balance of workload among processors, and at the same time minimizes the communication requirements by various modules of the code. Our code is based on the Hénon MC method, with algorithmic modifications including a parallel random number generation scheme, and a parallel sorting algorithm. We presented the first collisional $N$-body simulations of star clusters with $N$ covering three orders of magnitude and reaching up to $N=10^{7}$. The corecollapse times obtained in our simulations are in good agreement with previous studies, providing basic validation of our code. We also tested our implementation on 1-1024 processors. The code scales linearly up to 64 processors for all cases considered, after which it saturates, which we find to be characteristic of the parallel sorting algorithm. The overall performance of the parallelization is impressive, delivering maximum speedups of up to $220 \times$ for $N=10^{7}$.

Interesting future lines of work may include reducing the communication overhead by overlapping communication with computation. In addition to the distributed memory parallel version, $\mathrm{CMC}$ has also an optional feature that accelerates parts of the algorithm using a general purpose GPU, described in the Appendix. An important next step toward reaching even higher $N$ values is the development of a hybrid code which can run on heterogeneous distributed architectures with GPUs. With progress along these lines, we may be able to reach the domain of galactic nuclei for the first time. Many galactic nuclei contain massive, dense star clusters, so-called nuclear star clusters, which are thought to be significantly linked to the evolution of their host galaxies (e.g., Böker 2010), and their properties might still reflect to some extent the details of the formation of the galaxy (Merritt 2010). In addition, with their much larger masses and escape velocities, galactic nuclei are likely to retain many more stellar-mass black holes than globular clusters, and, thus, might significantly contribute to the black hole binary merger rate, as well as to the gravitational wave detection rate of advanced LIGO (Miller \& Lauburg 2009). Therefore, the study of galactic nuclei with a fully self-consistent dynamical code such as CMC has the potential to make strong predictions for future gravitational wave detection missions, and might give further insights into the evolution of galaxies.

This work was supported by NASA Grant NNX09A036G to F.A.R. We also acknowledge partial support from NSF Grants PHY05-51164, CCF-0621443, OCI-0724599, CCF-0833131, CNS-0830927, IIS- 0905205, OCI-0956311, CCF-0938000, CCF-1043085, CCF-1029166, and OCI-1144061, and from DOE Grants DE-FC02-07ER25808, DE-FG02-08ER25848, DE-SC0001283, DE-SC0005309, and DE-SC0005340. This research used resources of the National Energy Research Scientific Computing Center, which is supported by the Office of Science of the U.S. Department of Energy under Contract No. DE-AC02-05CH11231.

\section{APPENDIX}

\section{GPU ACCELERATION OF THE ORBIT COMPUTATION}

An optional feature of the CMC code is the GPU acceleration of the orbit computation that consists of finding $r_{\min }$ and $r_{\max }$ of each stellar orbit and sampling a new orbital position (see Section 2). As we have shown, the time complexity of both parts is $\mathcal{O}(N \log N)$, and the orbit and new position for each star can be determined independently from the other stars. This makes the orbit computation particularly well suited to being calculated on a GPU, not only because of the inherent parallelism of the algorithm, but also for the large number of memory accesses, which also scale as $\mathcal{O}(N \log N)$, and, thus, allow us to take advantage of the fast GPU memory.

Based on the structure of the algorithm, our implementation assigns one thread on the GPU to do the computations for one star. This ensures minimal data dependency between the threads since the same set of operations is performed on different data, and makes the bisection method and rejection technique implementations naturally suited for Single Instruction, Multiple Data architectures, such as the GPU. In the following we describe the specific adaptations of the serial implementation of the algorithms to the GPU architecture and present performance results.

\section{A.1. Memory Access Optimization}

To harness the high computation power of the GPU, it is very essential to have a good understanding of its memory hierarchy in order to develop strategies that reduce memory access latency. The first step toward optimizing memory accesses is to ensure that memory transfer between the host and the GPU is kept to a minimum. Another important factor that needs to be considered is global memory coalescing in the GPU which could cause a great difference in performance. When a GPU kernel accesses global memory, all threads in groups of a half-warp access a bank of memory at the same time (Nvidia 2010). Coalescing of memory accesses happens when data requested by these groups of threads are located in contiguous memory addresses, in which case they can be read in one (or very few number of) access(es). Hence, whether data are coalesced or not have a significant impact on an application's performance as it determines the degree of memory access parallelism. In CMC, the physical properties of each star are stored in a $\mathrm{C}$ structure, containing 46 double precision variables. The $N$ stars are stored in an array of such $\mathrm{C}$ structures.

Figure 10 gives a schematic representation of the data reorganization. At the top, the original data layout is shown, i.e., an array of structures. The kernels we parallelize only 


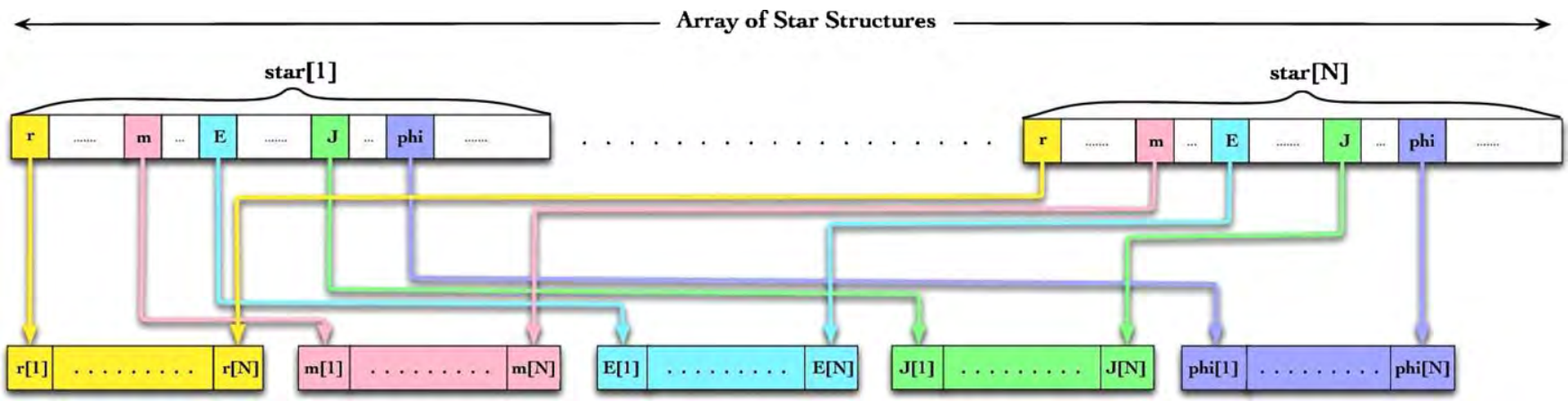

Figure 10. Data coalescing strategy used to strip the original star data structure and pack into contiguous arrays before transferring them to the GPU. The original data layout is an array of structures (top), which is reorganized into fewer separated arrays (bottom) for the variables used in the new orbits computation.

(A color version of this figure is available in the online journal.)

require 5 among the 46 variables present in the star structure: radial distance, $r$, mass $m$, energy, $E$, angular momentum, $J$, and potential at $r, \phi$, which are shown in different color. To achieve coalesced memory accesses, we need to pack the data before transferring it to the GPU in a way that they would be stored in contiguous memory locations in the GPU global memory. A number of memory accesses involve the same set of properties for different stars being accessed together by these kernels since one thread works on the data of one star. Hence, we extract and pack these into separate, contiguous arrays, one for each property. This ensures that the memory accesses in the GPU will be mostly coalesced. Also, by extracting and packing only the five properties required by the parallel kernels, we minimize the data transfer between the CPU and GPU.

\section{A.2. Random Number Generation}

For the generation of random numbers we use the same combined Tausworthe generator and parallel implementation scheme as described in Section 3.6. That is, for each thread that samples the position of a star, there is one random stream with an initial state that has been obtained by jumping multiple times in a seeded random sequence to ensure statistical independence between streams. As we will see later, to achieve optimal performance, $6000-8000$ threads, and, thus, streams, are required. This can be easily accommodated by one random sequence, as for our jump distance of $2^{80}$ and a random number generator period of $\approx 2^{113}, \approx 10^{10}$ non-overlapping streams can be generated. Once generated on the host, the initial stream states are transferred to the GPU global memory. Each thread reads the respective starting state from the memory and produces random numbers independently.

\section{A.3. Performance Results}

All our simulations are carried out on a $2.6 \mathrm{GHz}$ AMD PhenomTM Quad-Core Processor with 2 GB of RAM per core and an NVIDIA GTX280 GPU, with 30 multiprocessors, and $1 \mathrm{~GB}$ of RAM. The algorithms have been written in the CUDA C language, and were compiled with the version 3.1 of the CUDA compiler. All computations are done in double precision, using the only double precision unit in each multiprocessor on the GTX280 GPU.

We collect the timing results for five simulation time steps of a single-mass cluster with a Plummer density profile, and sizes ranging from $10^{6}$ to $7 \times 10^{6}$ stars, encompassing $\approx 25 \%$ of all globular cluster sizes (e.g., McLaughlin \& van der Marel 2005).

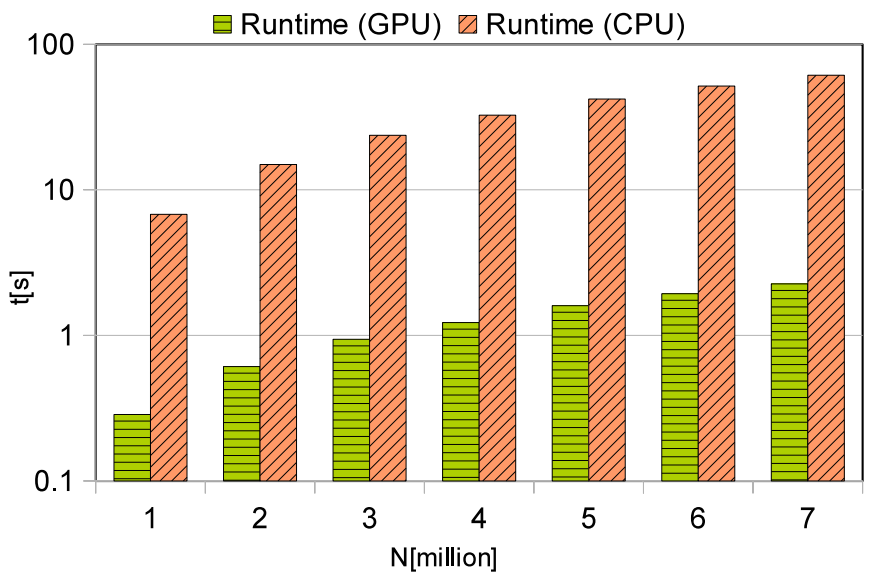

Figure 11. Comparison of total runtimes of the sequential and parallelized kernels for various $N$.

(A color version of this figure is available in the online journal.)

Figure 11 compares the GPU and CPU runtimes. Figure 12 shows the speedup of the new orbits computation part and the bisection and rejection kernels individually. All speedup values are with respect to the code performance on a single CPU. We see that the average speedups for the rejection and bisection kernels are 22 and 31, respectively. This is due to the difference in the number of floating point operations between the two kernels which is a factor of 10 . This makes a major difference on the CPU but not on the GPU as it has more arithmetic logic units. Indeed, the bisection and rejection kernels take about equal amount of time on the GPU for all $N$. This also indicates that the performance of these kernels is only limited by the memory bandwidth as they roughly require the same amount of global memory accesses.

We also observe that the total speedup increases slightly as the data size increases.

In general, we obtain very good scalability. Analyzing the dependence of the runtime on $N$ in Figure 11 we find that the GPU runtimes follow closely the kernel's complexity of $\mathcal{O}(N \log N)$. The runtimes on the CPU, on the other hand, have a steeper scaling with $N$, such that the run with $N=7 \times 10^{6}$ takes a factor of 11 longer than with $N=10^{6}$, instead of the expected factor of eight. The reason for the somewhat worse scaling of the runtime on the CPU is not yet clear and remains to be investigated in the future.

Note that as the memory transfer between the CPU and GPU is currently not optimized, our speedup calculations do not 


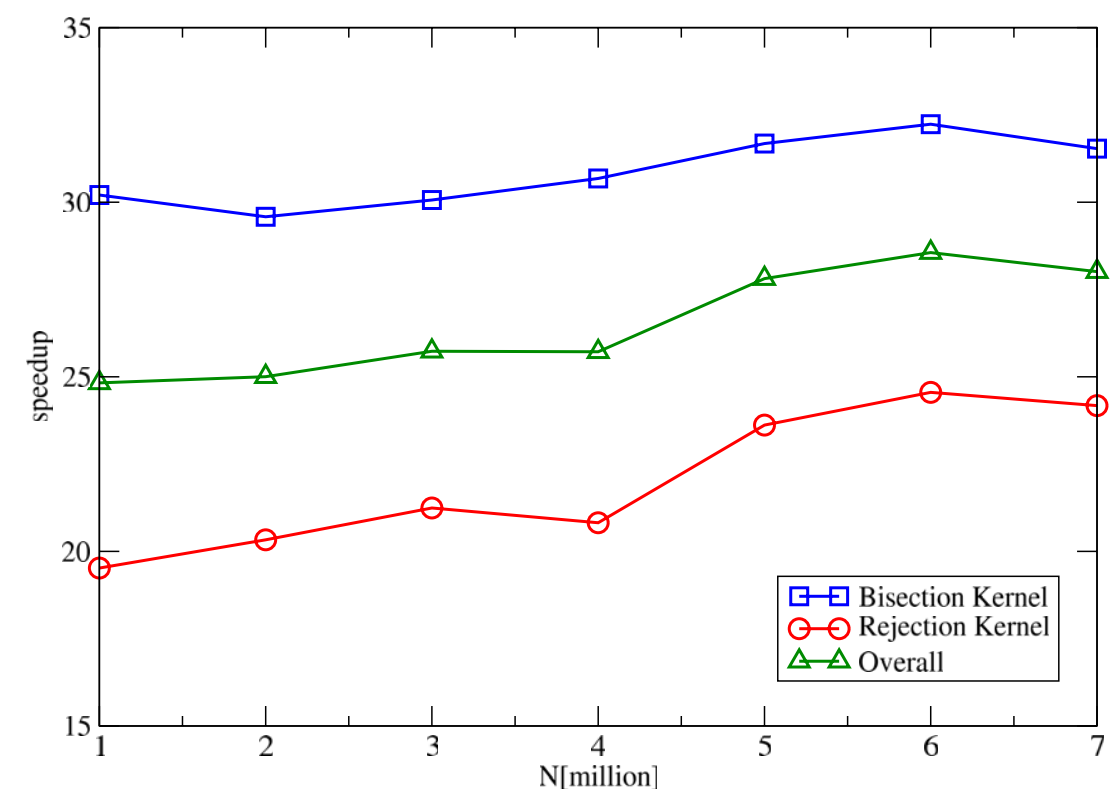

Figure 12. Overall and individual speedups of the bisection and rejection kernels. The mean overall speedup was observed to be $28 \times$, with the individual mean speedups being $22 \times$ and $31 \times$ for the bisection and rejection kernels, respectively.

(A color version of this figure is available in the online journal.)

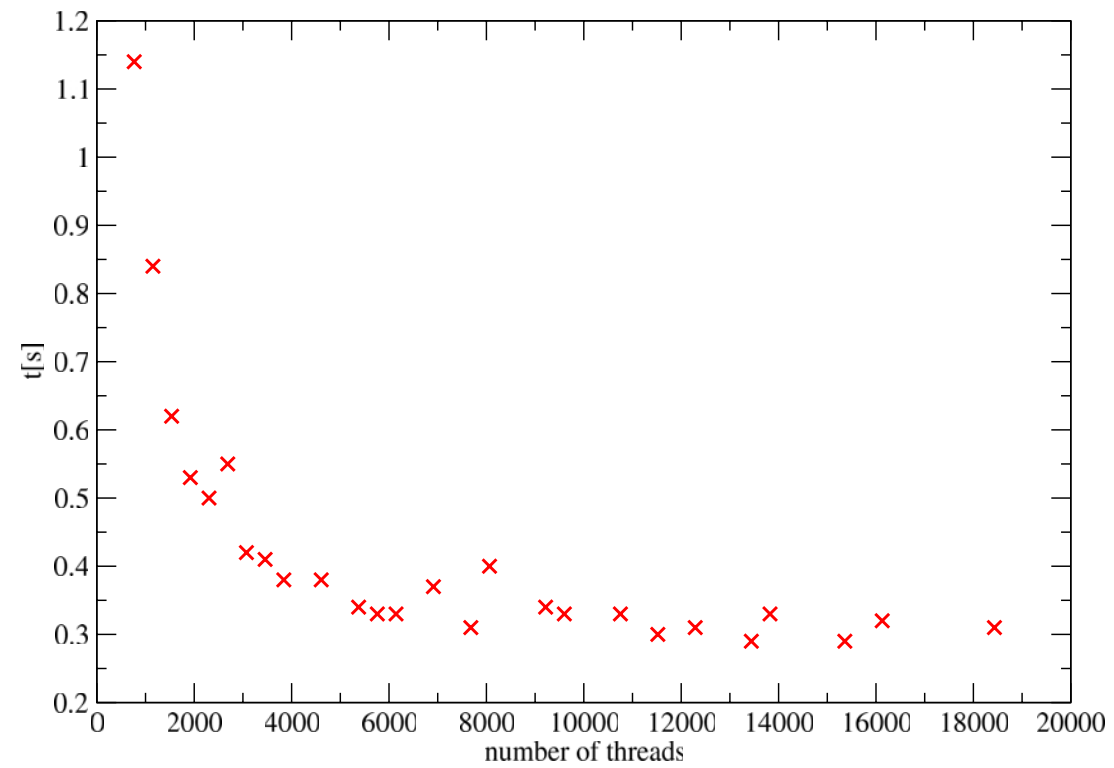

Figure 13. Total runtime of all kernels over the total number of threads. The runtime decreases with increasing thread number and saturates at around 6000 threads, which is expected to be due to the finite memory bandwidth.

(A color version of this figure is available in the online journal.)

include that overhead. However, as we transfer only a subset of the entire data for each star, there is the potential space for improvement to interleave kernel computations with data transfer and substantially reduce this overhead.

Finally, we looked at the influence of GPU-specific parameters on the runtime. In order to improve performance and utilization of the 30 multiprocessors, we partitioned our data space into a one-dimensional grid of blocks on the GPU. Due to the complexity of the expressions involved in the calculations of orbital positions, our kernels use a significant amount of registers (64 registers per thread). Thus, the block dimension is restricted to 256 threads per block as the GTX280 GPU has only 16,384 registers per block. To analyze the performance, we first made a parameter scan in the block and grid dimensions by varying the block sizes from 64 to 256 and the grid sizes from 12 to 72 . Figure 13 shows the total runtime of all kernels as a function of the total number of active threads on the GPU. As expected, the runtime decreases with increasing thread number but saturates at around 6000 threads. The saturation is most likely due to the finite memory bandwidth, as we noted before that the runtime of the kernels is mainly determined by the number of memory accesses as opposed to the number of floating point operations. One can also see that the curve shows little scatter, $\approx 0.1 \mathrm{~s}$, which means that the specific size of a single block of threads has a minor effect on performance. We furthermore find that for a given total number of threads, the runtime is shortest when the total number of thread blocks is a multiple of the number of multiprocessors, in our case 30 . 


\section{REFERENCES}

Binney, J., \& Tremaine, S. 2008, Galactic Dynamics (2nd ed.; Princeton, NJ: Princeton Univ. Press)

Böker, T. 2010, in IAU Symp. 266, Star Clusters: Basic Galactic Building Blocks throughout Time and Space, ed. R. de Grijs \& J. Lepine (Cambridge: Cambridge Univ. Press), 58

Casertano, S., \& Hut, P. 1985, ApJ, 298, 80

Chatterjee, S., Fregeau, J. M., Umbreit, S., \& Rasio, F. A. 2010, ApJ, 719, 915

Cohn, H. 1980, ApJ, 242, 765

Collins, J. C. 2008, Army Research Laboratory, 4, 41

Fraser, W. D., \& McKellar, A. C. 1970, JACM, 17, 496

Fregeau, J. M., Cheung, P., Portegies Zwart, S. F., \& Rasio, F. A. 2004, MNRAS, 352,1

Fregeau, J. M., Gürkan, M. A., Joshi, K. J., \& Rasio, F. A. 2003, ApJ, 593, 772

Fregeau, J. M., \& Rasio, F. A. 2007, ApJ, 658, 1047

Freitag, M., \& Benz, W. 2001, A\&A, 375, 711

Giersz, M. 1998, MNRAS, 298, 1239

Giersz, M., Heggie, D. C., Hurley, J., \& Hypki, A. 2011, arXiv:1112.6246

Goswami, S., Umbreit, S., Bierbaum, M., \& Rasio, F. A. 2012, ApJ, 752, 43

Gropp, W., Lusk, E., \& Skjellum, A. 1994, Using MPI: Portable Parallel Programming with the Message Passing Interface (Cambridge, MA: MIT Press)

Heggie, D., \& Hut, P. (ed.) 2003, The Gravitational Million-Body Problem: A Multidisciplinary Approach to Star Cluster Dynamics (Cambridge: Cambridge Univ. Press)

Heggie, D. C., \& Giersz, M. 2009, MNRAS, 397, L46
Heggie, D. C., \& Stevenson, D. 1988, MNRAS, 230, 223

Hénon, M. H. 1971, Ap\&SS, 14, 151

Hoare, C. A. R. 1961, Commun. ACM, 4, 321

Hurley, J. R., Pols, O. R., \& Tout, C. A. 2000, MNRAS, 315, 543

Hurley, J. R., \& Shara, M. M. 2012, MNRAS, 425, 2872

Hurley, J. R., Tout, C. A., \& Pols, O. R. 2002, MNRAS, 329, 897

Hut, P., Makino, J., \& McMillan, S. 1988, Natur, 336, 31

Jalali, B., Baumgardt, H., Kissler-Patig, M., et al. 2012, A\&A, 538, A19

Joshi, K. J., Nave, C. P., \& Rasio, F. A. 2001, ApJ, 550, 691

Joshi, K. J., Rasio, F. A., \& Portegies Zwart, S. 2000, ApJ, 540, 969

L'Ecuyer, P. 1999, MaCom, 68, 261

Li, X., Lu, P., Schaeffer, J., et al. 1993, ParC, 19, 1079

Lightman, A. P., \& Shapiro, S. L. 1978, RvMP, 50, 437

Lusk, E., Doss, N., \& Skjellum, A. 1996, ParC, 22, 789

McLaughlin, D. E., \& van der Marel, R. P. 2005, ApJS, 161, 304

Merritt, D. 2010, ApJ, 718, 739

Miller, M. C., \& Lauburg, V. M. 2009, ApJ, 692, 917

Nvidia. 2010, http://developer.download.nvidia.com, 1

Spitzer, L. 1987, Dynamical Evolution of Globular Clusters (Princeton, NJ: Princeton Univ. Press), 191

Stodolkiewicz, J. S. 1982, AcA, 32, 63

Trenti, M., Vesperini, E., \& Pasquato, M. 2010, ApJ, 708, 1598

Umbreit, S., Fregeau, J. M., Chatterjee, S., \& Rasio, F. A. 2012, ApJ, 750,31

Zonoozi, A. H., Küpper, A. H. W., Baumgardt, H., et al. 2011, MNRAS, 411, 1989 